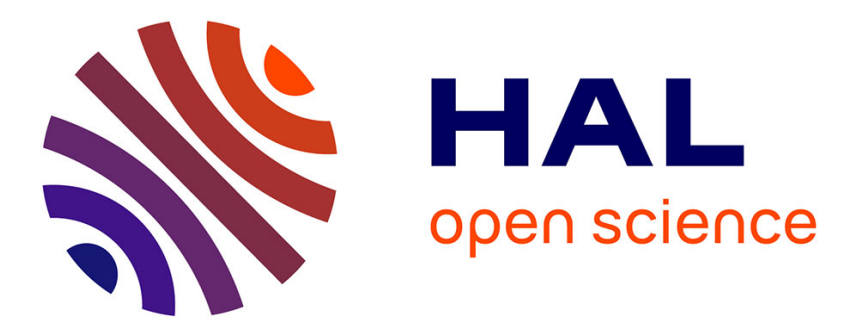

\title{
Simulation of the discharge propagation in a capillary tube in air at atmospheric pressure
}

\author{
Jaroslav Jánsky, Fabien Tholin, Zdeněk Bonaventura, Anne Bourdon
}

\section{To cite this version:}

Jaroslav Jánsky, Fabien Tholin, Zdeněk Bonaventura, Anne Bourdon. Simulation of the discharge propagation in a capillary tube in air at atmospheric pressure. Journal of Physics D: Applied Physics, 2010, 43 (39), pp.395201. 10.1088/0022-3727/43/39/395201 . hal-00569716

\section{HAL Id: hal-00569716 https://hal.science/hal-00569716}

Submitted on 25 Feb 2011

HAL is a multi-disciplinary open access archive for the deposit and dissemination of scientific research documents, whether they are published or not. The documents may come from teaching and research institutions in France or abroad, or from public or private research centers.
L'archive ouverte pluridisciplinaire HAL, est destinée au dépôt et à la diffusion de documents scientifiques de niveau recherche, publiés ou non, émanant des établissements d'enseignement et de recherche français ou étrangers, des laboratoires publics ou privés. 


\title{
Simulation of the discharge propagation in a capillary tube in air at atmospheric pressure
}

\author{
Jaroslav Jánský ${ }^{1}$, Fabien Tholin ${ }^{1}$, Zdeněk Bonaventura ${ }^{1,2}$ and \\ Anne Bourdon ${ }^{1}$ \\ ${ }^{1}$ Ecole Centrale Paris, EM2C Laboratory, UPR 288 CNRS, Grande voie des vignes, \\ 92295 Châtenay-Malabry Cedex, France \\ 2 Masaryk University, Faculty of Science, Department of Physical Electronics, \\ Kotlářská 2, 61137 Brno, Czech Republic \\ E-mail: jaroslav.jansky@em2c.ecp.fr \\ PACS numbers: 52.25.Os, 52.25.Dg, 52.25.Fi, 52.40.Hf, 52.65.-y, 52.80.Hc, 52.80.Tn, \\ 82.33.Xj
}

Keywords: streamer discharge, dielectric capillary tube, photoemission

Submitted to: J. Phys. D: Appl. Phys.

20 July 2010 


\begin{abstract}
This paper presents simulations of an air plasma discharge at atmospheric pressure initiated by a needle anode set inside a dielectric capillary tube. We have studied the influence of the tube inner radius and its relative permittivity $\varepsilon_{\mathrm{r}}$ on the discharge structure and dynamics. As a reference, we have used a relative permittivity $\varepsilon_{\mathrm{r}}=1$ to study only the influence of the cylindrical constraint of the tube on the discharge. For a tube radius of $100 \mu \mathrm{m}$ and $\varepsilon_{\mathrm{r}}=1$, we have shown that the discharge fills the tube during its propagation and is rather homogeneous behind the discharge front. When the radius of the tube is in the range 300 to $600 \mu \mathrm{m}$, the discharge structure is tubular with peak values of electric field and electron density close to the dielectric surface. When the radius of the tube is larger than $700 \mu \mathrm{m}$, the tube has no influence on the discharge which propagates axially. For a tube radius of $100 \mu \mathrm{m}$, when $\varepsilon_{\mathrm{r}}$ increases from 1 to 10 , the discharge structure becomes tubular. We have noted that the velocity of propagation of the discharge in the tube increases when the front is more homogeneous and then, the discharge velocity increases with the decrease of the tube radius and $\varepsilon_{\mathrm{r}}$. Then, we have compared the relative influence of the value of tube radius and $\varepsilon_{\mathrm{r}}$ on the discharge characteristics. Our simulations indicate that the geometrical constraint of the cylindrical tube has more influence than the value of $\varepsilon_{\mathrm{r}}$ on the discharge structure and dynamics. Finally, we have studied the influence of photoemission processes on the discharge structure by varying the photoemission coefficient. As expected, we have shown that photoemission, as it increases the number of secondary electrons close to the dielectric surface, promotes the tubular structure of the discharge.
\end{abstract}

\title{
1. Introduction
}

In the past decade, there has been an increasing interest for systems and processes combining atmospheric pressure plasma discharges with catalysts for different applications as the treatment of polluted air flows containing traces of VOCs (Volatile Organic Compounds) or nitrogen oxides [Hammer, 2002; Pasquiers, 2004; Kim, 2004]. The plasma-catalyst coupling requires to use catalyst supports as pellets, monoliths, foams or porous media. Then in these processes, atmospheric pressure discharges are produced in spatially confined geometries as microcavities and pores of the considered materials. However, only few studies have been performed on the physics of a discharge in contact with a porous material [Kim, 2004; Blin-Simiand et al., 2005; Guaitella et al., 2006; Sato et al., 2009; Hensel et al., 2007]. It is interesting to note that recently, Hensel [2009] showed that in microporous ceramic foams the pore size and the amplitude of the applied voltage were critical parameters for the discharge structure. If the pore size and voltage are small, a surface dielectric barrier discharge is observed but, above a certain voltage threshold, the discharge occurs inside the ceramics.

Atmospheric pressure plasmas are also routinely used to functionalize surfaces of polymers with surface roughness which can range from hundreds of nanometers to tens of $\mu \mathrm{m}$. Bhoj and Kushner [2008] have simulated the treatment by a repetitively pulsed discharge of humid air of a rough polypropylene surface having micrometer-sized strandlike features. These authors showed that the penetration of charged species into the rough surface depends on the discharge polarity. In negative discharges, the penetration 
of electrons into surface structures is limited, which locally produce reactive species by electron impact. In positive discharges, a sheath like region near the surface prevents electrons to penetrate into the surface and therefore there is less local production of radicals.

Recently, spatially confining the plasma to dimensions of $1 \mathrm{~mm}$ or less has also appeared to be a promising approach to the generation of stable, glow discharges at atmospheric pressure for a wide range of applications. Many studies are carried out on different types of so-called microdischarges [Becker et al., 2006, and references therein] as microhollow cathode discharge (MHCD), capillary plasma electrode (CPE) and plasma jets [Walsh et al., 2010]. Microdischarges can be generated in different gases and many studies have been carried out with rare gases. Despite the numerous experiments on microdischarges, some basic mechanisms of these discharges are still poorly understood as for example the propagation of atmospheric pressure plasma jets which have been demonstrated to be discrete fast-moving plasma packets or bullets [Teschke et al., 2005; Lu and Laroussi, 2006; Sands et al., 2008; Shi et al., 2008].

In this work, as a first step to better understand atmospheric pressure discharge phenomena in porous materials, we propose to study an air plasma discharge at atmospheric pressure in a constrained geometrical configuration: a plasma discharge initiated by a needle inside a dielectric capillary tube (i.e. tube with a typical inner radius of the order of $100 \mu \mathrm{m})$. It is important to note that in parallel to our numerical studies, an experimental study is underway at the LPGP laboratory in Orsay (France). The detailed comparison with experiment is beyond the scope of this paper and will be presented in a separate dedicated paper.

To study the influence of the confinement of the discharge by the capillary tube, a first important point is the distance between the dielectric surface and the point of initiation of the discharge. Indeed, Allen et al. [Allen and Mikropoulos, 1999; Tan et al., 2007] showed that, close to dielectric surfaces, discharges in air usually propagate in two modes: a fast component over the dielectric surface and a slow one in ambient air. It is important to note that in all these studies, the discharge is generated in a point-to-plane geometry and the dielectric is a plane or a cylinder set on one side of the discharge. Recently a pin-pin electrode geometry has been used by Sobota et al. [2009] in pure Argon to study the discharge propagation over a flat dielectric surface and in the gas close to the dielectric. These authors also concluded that the discharge propagates faster over the dielectric surface than through the gas. It is important to note that in both studies the electrodes were in contact [Allen and Mikropoulos, 1999] or at a few millimeters from the dielectric surface [Tan et al., 2007; Sobota et al., 2009]. In this work, we propose to study discharge initiation and propagation in capillary tubes and therefore to study the influence of a cylindrical constraint on the discharge. Furthermore, as we use capillary tubes, inner radii of tubes are of the order of $100 \mu \mathrm{m}$ which corresponds to the order of typical radii of streamer discharges in air at atmospheric pressure. Then in this work, we propose to vary the radius of the capillary tube to study the influence of surface and volume processes on the discharge structure and dynamics. 
It is important to mention that only few works have been devoted to the study of the influence of the value of the relative permittivity $\varepsilon_{\mathrm{r}}$ of dielectric materials on discharge properties. In surface dielectric barrier discharges in air, Gibalov and Pietsch [2000] have shown that when $\varepsilon_{\mathrm{r}}$ decreases from 9.8 to 2.4 the surface dielectric barrier discharge propagates farther on the surface. In the work of Sobota et al. [2009] in Argon, for $\varepsilon_{\mathrm{r}}$ varying between $2.3-4.5$ and 9.1 , a very small influence of the surface discharge velocity is observed. Babaeva et al. [Babaeva et al., 2006; Babaeva and Kushner, 2009] studied the influence of dielectric dust particles on the discharge structure in air at atmospheric pressure. They showed that streamers encountering a particle could branch or initiate new streamers depending on the permittivity and size of the particles. For small solid particles with low permittivity, the plasma envelops the particle and may produce branching. Particles with higher permittivity stop and reinitiate the streamer. Georghiou et al. [2005] have carried out simulations in atmospheric air in a needle-toplane configuration with a dielectric tube. The needle is set outside of the tube and the discharge is initiated close to the needle and then enters in the tube. Georghiou et al. [2005] studied the influence of $\varepsilon_{\mathrm{r}}$ variations in the range 2.2 to 6.2 for a tube of $200 \mu \mathrm{m}$ of radius and they showed that the velocity of the discharge increases as $\varepsilon_{\mathrm{r}}$ increases. Then in this work we propose to carry out a parametric study on the value of $\varepsilon_{\mathrm{r}}$ of the tube to study its influence on the discharge structure. As a reference, we consider the case $\varepsilon_{\mathrm{r}}=1$ which allows to study only the geometrical constraint on the discharge dynamics due to the presence of the tube. Then we have considered values of $\varepsilon_{\mathrm{r}}$ in the range 2 to 10 to represent different material permittivities (e.g. for PETG $\varepsilon_{\mathrm{r}}=2.4$ and for alumine oxide $\varepsilon_{\mathrm{r}}=9.1$ ).

Finally, the interaction of the discharge with a dielectric surface requires to study secondary processes at the dielectric surface. In most simulations of surface dielectric barrier discharges, secondary electron emissions due to ion bombardment are considered. However, electrons may be also emitted from other processes as field emission or secondary emission of electrons by impact of metastable molecules. For all these processes, it is important to note that secondary emission coefficients are still poorly known. Electrons may also be emitted from detachment of negative ions, or electrons on the dielectric surface, left over by previous discharges. In this work, we have considered that no charges are deposited on the dielectric surface at the beginning of the simulation. Finally, electrons can be emitted from the dielectric surface by photoemission. In this work, we have assumed that the flux of electrons emitted from the surface is proportional to the flux of photons at the surface [Potin, 2001; Georghiou et al., 2001; Hallac et al., 2003]. In this case, to model the photoemission source term it is necessary to compute a volume integral and to determine a photoemission coefficient. In fact, the value of this coefficient is poorly known in air and then in many studies of dielectric barrier discharges in air, photoemission is often neglected [e.g. Unfer and Boeuf, 2009]. In this work, we propose to carry out simulations without photoemission and then to take it into account with different values of the photoemission coefficient to study its influence 
on the discharge dynamics.

In Section 2, we present the studied discharge set-up and the discharge model. In Section 3.1, first we present discharge simulations in the needle-to-plane geometry without dielectric tube. Then the discharge propagating in a dielectric capillary tube of radius $R_{\text {tube }}=100 \mu \mathrm{m}$ with $\varepsilon_{\mathrm{r}}=1$ is considered. In Section 3.3, the permittivity of the tube is varied and in Section 3.4 the inner radius of the tube is varied. Finally in Section 3.5 the influence of photoemission processes on the discharge are discussed.

\section{Model formulation}

\subsection{Studied configuration}

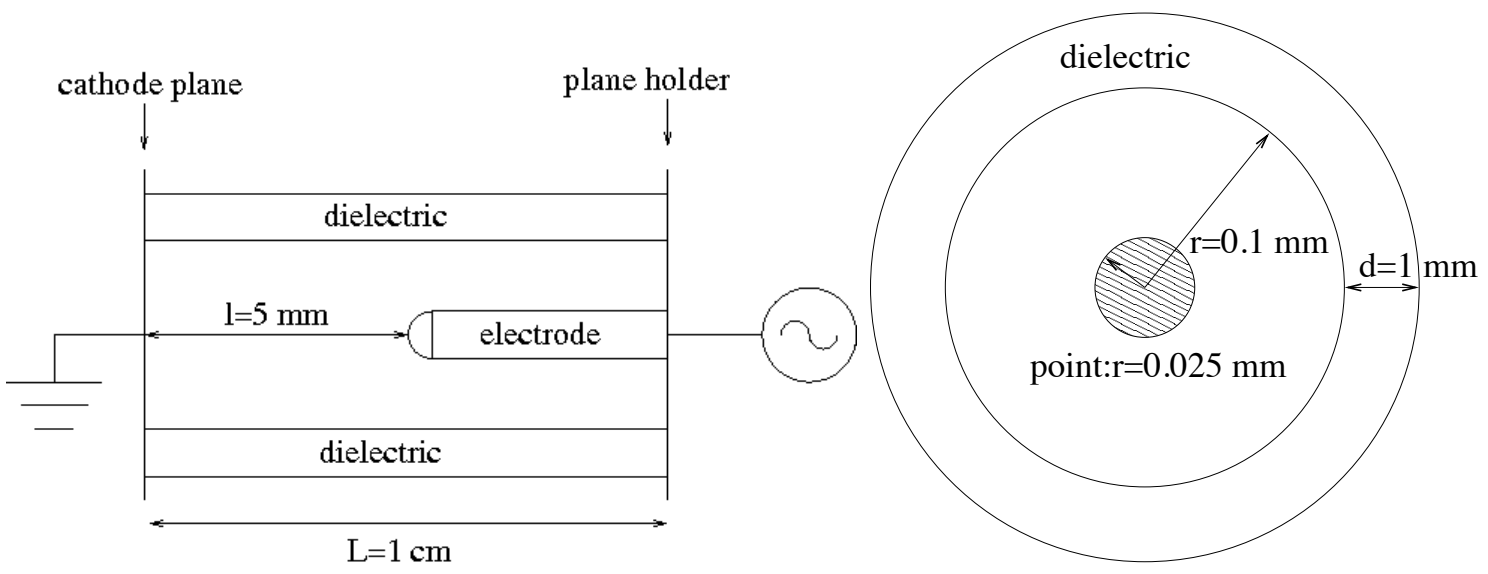

Figure 1. Sideview and topview schematics of discharge set-up for a capillary tube with an inner radius of $100 \mu \mathrm{m}$.

The discharge set-up is shown on Figure 1. A metallic point anode on a metallic plane holder is set at $5 \mathrm{~mm}$ from a metallic cathode plane. The tip of the point is a semisphere with a radius of curvature of $25 \mu \mathrm{m}$. The point is immersed in a $1 \mathrm{~cm}$ long dielectric capillary tube. The inner radius of the tube varies between $R_{\text {tube }}=100$ and $700 \mu \mathrm{m}$ and the thickness of the tube is $1 \mathrm{~mm}$. In the experiments at LPGP, the length of the tube and then the interelectrode gap may vary from 5 up to $15 \mathrm{~mm}$, and as a first step in this work, we have only considered an interelectrode gap of $5 \mathrm{~mm}$. In the experiments, the applied voltage is a single positive high voltage square pulse of 6 to $18 \mathrm{kV}$ applied at the tip. In this work, as a first step, we have considered that the voltage is constant and applied at the beginning of the discharge simulation and, we have studied voltages in the range $6-9 \mathrm{kV}$. It is important to note that no gas heating was observed in the experiments.

\subsection{Discharge model}

A classical fluid model based on the drift-diffusion equations for electrons and ions coupled with the Poisson's equation [e.g. Kulikovsky, 1997] is used to simulate the 
discharge propagation. To take into account the different dielectric permittivities in air and in the dielectric tube, we use Poisson's equation with variable coefficients [Unfer and Boeuf, 2009]:

$$
\begin{aligned}
& \frac{\partial n_{\mathrm{e}}}{\partial t}-\nabla \cdot\left(n_{\mathrm{e}} \mu_{\mathrm{e}} \mathbf{E}\right)-\nabla \cdot\left(D_{\mathrm{e}} \nabla n_{\mathrm{e}}\right)=S_{\mathrm{ph}}+S_{\mathrm{e}}^{+}-S_{\mathrm{e}}^{-}, \\
& \frac{\partial n_{\mathrm{p}}}{\partial t}+\nabla \cdot\left(n_{\mathrm{p}} \mu_{\mathrm{p}} \mathbf{E}\right)=S_{\mathrm{ph}}+S_{\mathrm{p}}^{+}-S_{\mathrm{p}}^{-}, \\
& \frac{\partial n_{\mathrm{n}}}{\partial t}-\nabla \cdot\left(n_{\mathrm{n}} \mu_{\mathrm{n}} \mathbf{E}\right)=S_{\mathrm{n}}^{+}-S_{\mathrm{n}}^{-}, \\
& \nabla \cdot(\varepsilon \nabla V)=-q_{\mathrm{e}}\left(n_{\mathrm{p}}-n_{\mathrm{n}}-n_{\mathrm{e}}\right)+\sigma \delta_{\mathrm{s}},
\end{aligned}
$$

where subscripts "e", "p" and "n" refer to electrons, positive and negative ions, respectively, $n_{i}$ is the number density of species $i, V$ is the potential, $\mathbf{E}=-\nabla V$ is the electric field, $\mu_{i}$ is the absolute value of the mobility of species $i$ and $D_{\mathrm{e}}$ is the diffusion coefficient of electrons. On timescales of interest for studies presented in this paper, diffusion of ions is neglected. $q_{\mathrm{e}}$ is the absolute value of electron charge, $\varepsilon=\varepsilon_{\mathrm{r}} \varepsilon_{0}$, $\varepsilon_{\mathrm{r}}$ and $\varepsilon_{0}$ are the permittivity of the studied medium, the relative permittivity and the permittivity of free space, respectively, and $\sigma \delta_{\mathrm{s}}$ represents the contribution of the charges deposited by the discharge on the dielectric surface. The $S^{+}$and $S^{-}$terms stand for the rates of production and loss of charged particles. In the present study the $S_{\mathrm{e}}^{+}$and $S_{\mathrm{p}}^{+}$production rates have meaning of the ionization rate due to the electron impact ionization of air molecules, which is denoted as $S_{\mathrm{i}}$ in the following. The $S_{\mathrm{i}}$ rate is defined in a standard fashion as $S_{\mathrm{i}}=n_{\mathrm{e}} \nu_{\mathrm{i}}$ where $\nu_{\mathrm{i}}=\alpha \mu_{\mathrm{e}}|\mathbf{E}|$ is the ionization frequency and $\alpha$ is the Townsend ionization coefficient. The $S_{\mathrm{ph}}$ term is the rate of electron-ion pair production due to photoionization in a gas volume.

Transport parameters and reaction rates of the model are assumed to be functions of the local reduced electric field $E / N$, where $E$ is the electric field magnitude and $N=2.45 \times 10^{25} \mathrm{~m}^{-3}$ is the air neutral density, and are taken from [Morrow and Lowke, 1997]. For the photoionization source term, the three-group $\mathrm{SP}_{3}$ model is used [Bourdon et al., 2007] with boundary conditions given in [Liu et al., 2007].

In this paper, axisymmetric discharges are studied and thus cylindrical coordinates $(x, r)$ are used with the $x$-axis as axis of the discharge. We have used a $1 \times 2 \mathrm{~cm}^{2}$ computational domain. The grid is Cartesian, with a mesh size of $1 \mu \mathrm{m}$ at the electrode tip on the axis. In the axial direction, the mesh size is expanded towards the cathode following a geometric progression until it reaches $5 \mu \mathrm{m}$ and then is kept constant. In the radial direction, when there is no dielectric tube, the mesh is expanded from 1 to $5 \mu \mathrm{m}$ and is kept fixed until $r=1 \mathrm{~mm}$. Beyond this region, the grid expands following a geometric progression. When there is a dielectric tube, the mesh is refined in the radial direction from $1 \mu \mathrm{m}$ at the axis to $0.5 \mu \mathrm{m}$ close to the dielectric surface and then expanded following a geometric expansion. As an example, the final rectilinear grid for the whole computational domain for the condition of Figure 1 with a capillary tube of $R_{\text {tube }}=100 \mu \mathrm{m}$ is a grid with $n_{x} \times n_{r}=1512 \times 300$ cells. The tip of the anode point is 
located at $x=0.5 \mathrm{~cm}$ and the grounded cathode is located at $x=0$.

Poisson's equation is discretized using a finite volume approach over the whole computational domain. At the metallic electrode-air interfaces, the ghost-fluid method is used [Célestin et al., 2009]. In the radial direction for both the symmetry axis and the border of the computational domain, homogeneous Neumann boundary conditions $(\nabla V \cdot \mathbf{n}=0, \mathbf{n}$ being the normal vector of the surface boundary) are used. Finally, the resulting linear system is solved using the direct solver MUMPS [Amestoy et al., 2001]. To reduce the memory requirements of the direct solver, it is important to take advantage of the fact that the obtained matrix is symmetric positive definite.

The charged species transport equations are solved using the improved ScharfetterGummel algorithm [Kulikovsky, 1995] with linear field approximation (ISG-1). The continuity equations are solved in the whole computational domain when no dielectric tube is considered and otherwise only inside the dielectric tube. It is important to note that the accuracy of the ISG-1 scheme depends on the value of a parameter $\epsilon_{\mathrm{ISG}}$ which has to be less than 1 . Indeed, when coefficient $\epsilon_{\mathrm{ISG}}$ changes from 0 to 1 , the scheme transforms from a vary accurate but dispersive one to a less accurate, diffusive, but monotonic Scharfetter - Gummel algorithm. In [Kulikovsky, 1995], Kulikovky states that the parameter $\epsilon_{\mathrm{ISG}}$ has to be chosen experimentally and based on his experience he recommends to use values in the range 0.01-0.04. In this work, we have used a standard value of $\epsilon_{\mathrm{ISG}}=0.01$ for all simulations except for the results presented in Sections 3.3 and 3.5 where a higher value of $\epsilon_{\mathrm{ISG}}=0.1$ has been used. The influence of the value of $\epsilon_{\mathrm{ISG}}$ on the results is discussed in Section 3.5. However, it is important to mention in this section that the increase of the value of $\epsilon_{\mathrm{ISG}}$ from 0.01 to 0.1 has a very small influence on the results presented in Sections 3.3 and 3.5, but allows to remove small oscillations that we have observed with lower values of $\epsilon_{\mathrm{ISG}}$.

To calculate the photoionization source term with the three-group $\mathrm{SP}_{3}$ model, we have also used the MUMPS direct solver [Amestoy et al., 2001]. It has been verified by practical tests that very accurate results for the photoionization production rate can be obtained even if the photoionization is calculated once during every five steps of the execution of the streamer model. This approach is justified due to the very small time step used in the streamer modeling. For other source terms in continuity equations, time integration is based on a 4th order Runge-Kutta method.

In this work, we have taken into account simplified boundary conditions for continuity equations at anode and cathode surfaces. On the cathode plane, the homogeneous Neumann conditions are applied for all charged particle fluxes. At the point anode, the positive ion fluxes are fixed equal to zero while the negative species fluxes are estimated using the homogeneous Neumann conditions. At the dielectric surface, negative ion fluxes are fixed equal to zero and electron flux from the dielectric surface is assumed to be due to secondary emission by ion bombardment and photoemission. For the secondary emission of electrons due to ion bombardment, we have tested different values of the secondary emission coefficient in the range $0.001-0.1$ and no visible differences on the discharge propagation velocity or maximum electric field have been observed. For all 
results presented in this work, we have used a value of 0.1 . The use of this high value is also a way to take into account roughly other secondary emission processes as secondary emission of electrons by impact of metastable molecules and field emission. For photoemission, the flux of electrons emitted from the surface is assumed to be proportional to the flux of photons at the surface [Potin, 2001; Georghiou et al., 2001; Hallac et al., 2003] :

$$
\mathbf{j}_{\text {photoem }} \cdot \mathbf{n}=\gamma_{\text {photoem }} \boldsymbol{\varphi} \cdot \mathbf{n},
$$

where $\mathbf{n}$ is the unit vector perpendicular to the dielectric surface, $\gamma_{\text {photoem }}$ is a coefficient and $\boldsymbol{\varphi}(\mathbf{r})$ is the photon flux at the dielectric surface in direction $\mathbf{r}$. As the diameter of the tube is small, we have assumed that the radiation emitted by the discharge which produces photoemission is not absorbed by the gas, then the photon flux at the dielectric surface at point of observation $\mathbf{r}$ due to source points emitting photons at $\mathbf{r}^{\prime}$ is

$$
\boldsymbol{\varphi}(\mathbf{r})=\iiint_{V^{\prime}} \frac{I\left(\mathbf{r}^{\prime}\right)\left(\mathbf{r}-\mathbf{r}^{\prime}\right)}{4 \pi\left|\mathbf{r}-\mathbf{r}^{\prime}\right|^{3}} \mathrm{~d} V^{\prime}
$$

In this model, to simplify calculations, the production of photons $I$ is assumed to be equal to the ionization production rate $S_{i}$ [Odrobina and Cernak, 1991; Georghiou et al., 2001; Brandeburg et al., 2009] and then the only coefficient to be determined to model the photoemission source term is $\gamma_{\text {photoem }}$ in Eq. (5). In fact, this coefficient is poorly known in air and then in many studies of dielectric barrier discharges in air, photoemission is often neglected [e.g. Unfer and Boeuf, 2009]. In this work, for the results presented in Section 3.2 to 3.4, photoemission is not included. In Section 3.5, we take into account photoemission processes with different values of $\gamma_{\text {photoem }}$ to study the influence of photoemission on the discharge dynamics. We have considered three values for $\gamma_{\text {photoem }}=5 \times 10^{-4}, 5 \times 10^{-3}$ and $1 \times 10^{-1}$. The value $5 \times 10^{-3}$ is used for photoemission in pure $\mathrm{N}_{2}$ [Brandeburg et al., 2009] and also in air [Georghiou et al., 2001] and we have used a value which is ten times less and an upper limit. For our studied configuration, we have to calculate with Eq. (6) the component of the flux of photons normal to the dielectric surface. In the cylindrical coordinate system $(x, r, \theta)$, this flux at a point $\left(x, r=R_{\text {tube }}\right)$ on the dielectric surface is given by:

$$
\varphi_{r}\left(x, r=R_{\text {tube }}\right)=\int_{0}^{L} \mathrm{~d} x^{\prime} \int_{0}^{R_{\text {tube }}} r^{\prime} \mathrm{d} r^{\prime} I\left(r^{\prime}, x^{\prime}\right) M_{\mathrm{ph}}\left(x, r^{\prime}, x^{\prime}\right)
$$

and the function $M_{\mathrm{ph}}$ is defined as

$$
M_{\mathrm{ph}}\left(x, r^{\prime}, x^{\prime}\right)=2 \int_{0}^{\pi} \frac{\left[R_{\text {tube }}-r^{\prime} \cos \left(\theta^{\prime}\right)\right] \mathrm{d} \theta^{\prime}}{4 \pi\left[\left(x^{\prime}-x\right)^{2}+r^{\prime 2}+R_{\text {tube }}^{2}-2 R_{\text {tube }} r^{\prime} \cos \left(\theta^{\prime}\right)\right]^{\frac{3}{2}}}
$$

The computation of the volume integral of Eqs. (7) and (8) is very time consuming and then to reduce computation time, first, we have precalculated the geometrical factor $M_{\mathrm{ph}}$. Second, we have used a coarser mesh to calculate the photoemission source term as in [Hallac et al., 2003]. This coarser mesh is created in joining $2 \times 2$ blocks of cells in regions where the mesh is uniform. We have checked that the use of the coarser mesh has no influence on the accuracy of the calculated photoemission fluxes for the results 
presented in this paper.

Finally, to define the time step of the simulation, we use the same approach as in [Bourdon et al., 2007]. In all the simulations presented in this work, to initiate the discharge, a low initial uniform density of seed charges $\left(10^{4} \mathrm{~cm}^{-3}\right)$ is considered in the interelectrode gap. We have varied this initial density by one order of magnitude and we have checked that this low value has no influence on the discharge propagation as the electrons produced by photoionization in the volume and secondary processes and photoemission at the dielectric surface rapidly exceed the initial electron density.

\section{Results}

\subsection{Reference simulation in the needle-to-plane geometry without dielectric tube}

In this section, we first present discharge simulations in the needle-to-plane configuration described in Figure 1 without dielectric tube. Figure 2 shows the time sequence of the distributions of the electron density and absolute values of the electric field for an applied voltage of $9 \mathrm{kV}$. During the first $3 \mathrm{~ns}$ the discharge is initiated in the high Laplacian field close to the sharp point and expands in $x$ and $r$ directions until it reaches approximately a radius of $1 \mathrm{~mm}$. This sphere-shaped region close to the point has been

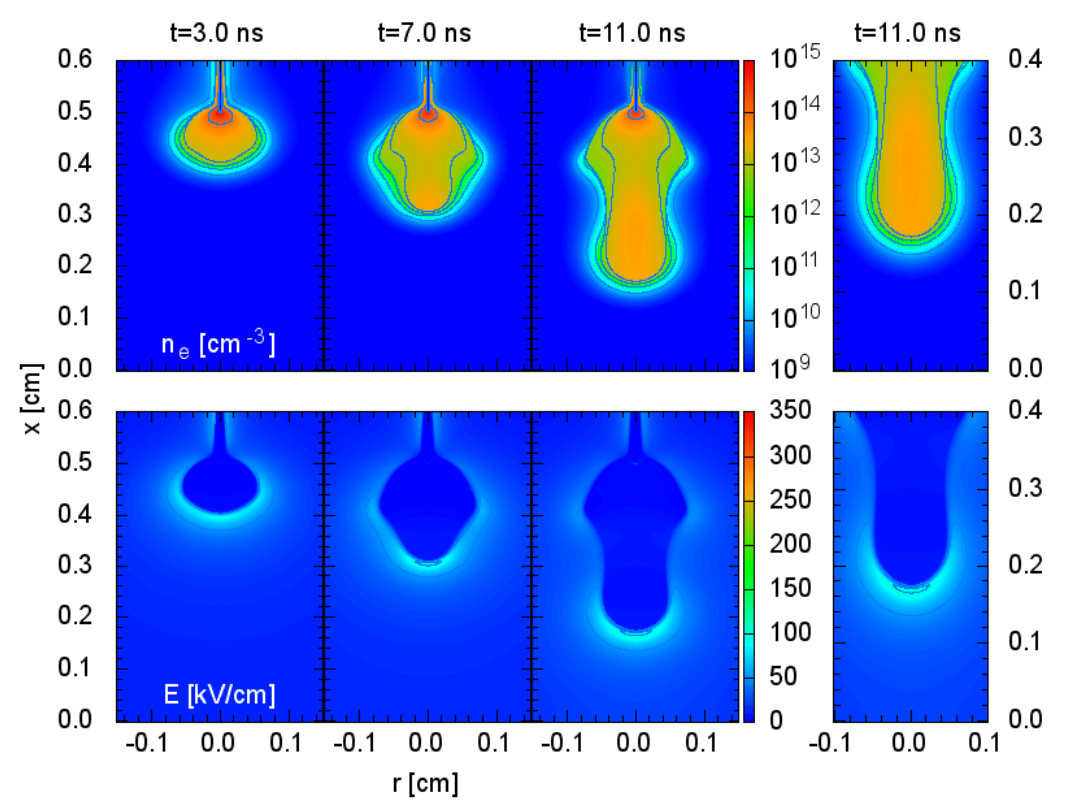

Figure 2. Propagation of the discharge in needle-to-plane geometry without capillary tube for an applied voltage of $9 \mathrm{kV}$. Cross-sectional views of the electron density and the magnitude of the electric field at $t=3,7$, and $11 \mathrm{~ns}$. Last figure of each line shows the discharge front in proportional scale at $t=11 \mathrm{~ns}$.

observed experimentally [e.g. Tardiveau et al., 2009] and numerically [e.g. Kulikovsky, 1998; Eichwald et al., 2008; Célestin et al., 2009] in different studies in point-to-plane geometry. After this first expansion phase, the discharge starts propagating on the axis. Between $t=7 \mathrm{~ns}$ and $t=11 \mathrm{~ns}$, the streamer head propagates with almost constant 
velocity and a maximum electric field of $E=110 \mathrm{kV} / \mathrm{cm}$ and a maximum electron density of $n_{\mathrm{e}}=3.5 \times 10^{13} \mathrm{~cm}^{-3}$. It is interesting to note that the discharge radius is of about $600 \mu \mathrm{m}$, which is about the radius of the largest capillary tube we have studied in this work. Then, the propagation of the discharge in tubes with radii less than $600 \mu \mathrm{m}$ is expected to change significantly the discharge dynamics. As the discharge approaches the cathode plane, its velocity slightly increases as will be shown in Figure 8 and finally, the positive streamer reaches the cathode at $t=13.8 \mathrm{~ns}$. It is important to note that the discharge propagation in this configuration is axial and stable. As part of preparatory work for the studies presented in this paper, we have carried out simulations with slightly different point geometries with the same radius of curvature at the tip, with and without the plane holder to test the influence of the geometry of the point on the results. In most simulations in point-to-plane configurations with interelectrode gaps of about 1 $\mathrm{cm}$, point electrodes are often assumed to be hyperboles [Kulikovsky, 1998], paraboles [Djermoune et al., 1995] or have a rather complex shape (e.g. a cylinder ending in a cone shape with a curved tip [Eichwald et al., 2008]). All these geometries guarantee a small axial Laplacian electric field (of about a few $\mathrm{kV} / \mathrm{cm}$ i.e. close to the stability field of $5 \mathrm{kV} / \mathrm{cm}$ at atmospheric pressure) far from the point. For simulations in non-uniform electric fields in long gaps as in [Babaeva and Naidis, 1996], a sphere electrode is used with an additional uniform electric field of a few $\mathrm{kV} / \mathrm{cm}$. In our studied configuration, the point electrode is a cylinder ended with a semisphere, and then, without the plane holder the Laplacian electric field on the axis decreases rapidly to zero. With the plane holder, a small axial Laplacian electric field is guaranteed far from the point on the axis and ensures a stable propagation of the discharge.

\subsection{Propagation of the discharge in a dielectric tube with $R_{\text {tube }}=100 \mu \mathrm{m}$ and permittivity $\varepsilon_{\mathrm{r}}=1$}

In this section, we present simulations of the discharge propagation in a capillary tube with an inner radius of $100 \mu \mathrm{m}$, the smallest radius we have considered in this work. To study first the influence of the radial geometrical constraint on the discharge dynamics, we use in this section, a permittivity of the tube of $\varepsilon_{\mathrm{r}}=1$. The influence of the value of $\varepsilon_{\mathrm{r}}$ will be discussed in Section 3.3. Figure 3 shows the time sequence of the distributions of the electron density and absolute values of the electric field for an applied voltage of $9 \mathrm{kV}$. The initiation phase of the discharge lasts less than $1 \mathrm{~ns}$ and then the discharge propagates in the tube with a rather planar discharge front. The proportional figures show that the electron density is uniform on the discharge front with maximum value of $n_{\mathrm{e}}=5.5 \times 10^{14} \mathrm{~cm}^{-3}$. The maximum of electric field $(E=300 \mathrm{kV} / \mathrm{cm})$ is located out of the axis close to the tube and the maximum of electric field on the axis is $E=220 \mathrm{kV} / \mathrm{cm}$. Between $t=1 \mathrm{~ns}$ and $5 \mathrm{~ns}$, the discharge front propagates with almost constant velocity. As the discharge approaches the cathode plane, its velocity slightly increases as will be shown in Figure 8 and finally, the discharge reaches the cathode at $t=5.7 \mathrm{~ns}$. In comparison to the case without the dielectric tube, we note that the 
Simulation of the discharge propagation in a capillary tube ...

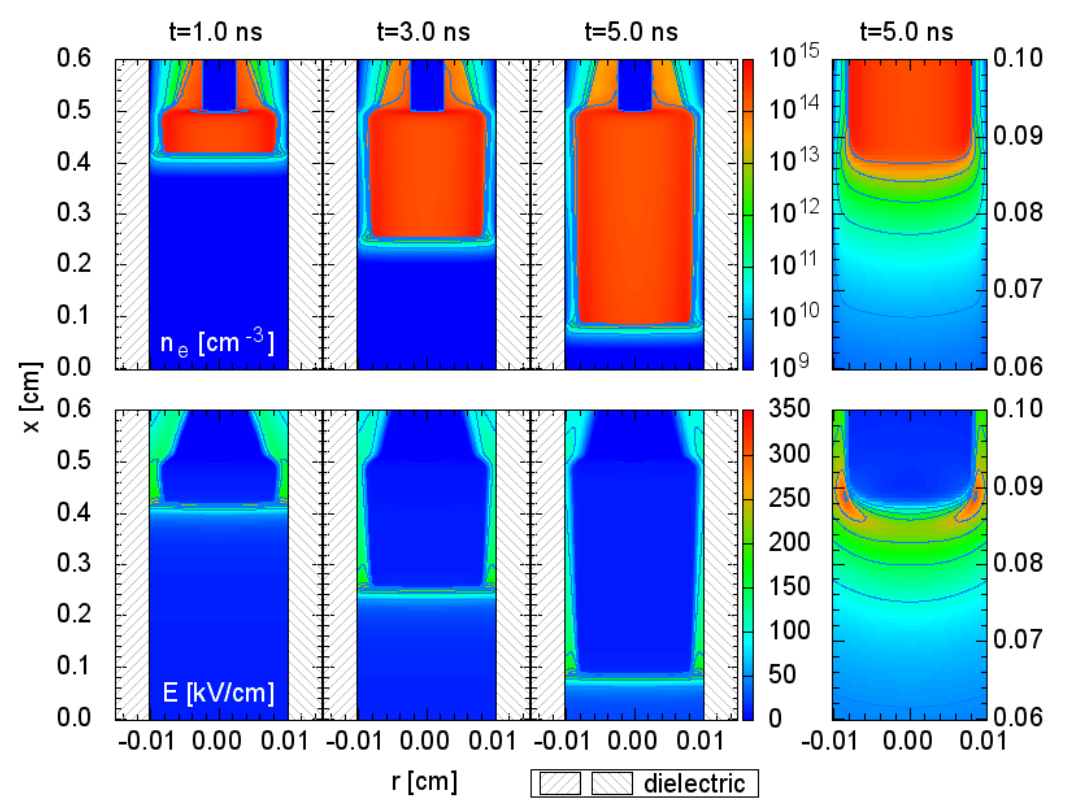

Figure 3. Propagation of the discharge in a capillary tube with $R_{\text {tube }}=100 \mu \mathrm{m}$ and permittivity $\varepsilon_{\mathrm{r}}=1$ for an applied voltage of $9 \mathrm{kV}$. Cross-sectional views of the electron density and the magnitude of the electric field at $t=1,3$, and $5 \mathrm{~ns}$. Last figure of each line shows the discharge front in proportional scale at $t=5 \mathrm{~ns}$.

initiation and the propagation phases are much faster with the capillary tube. The maximum electron density and electric field are both higher with the tube than without (16 and 3 times respectively). It is interesting to note on Figure 3 that the discharge is filling the whole tube during its propagation and is rather homogeneous. This is very interesting for applications, and then, we have also carried out simulations at another voltage of $6 \mathrm{kV}$ to study the influence of the voltage on the discharge structure. Figure 4 shows the time sequence of the distributions of the electron density and absolute values of the electric field for $6 \mathrm{kV}$. The discharge structure is similar to the one obtained for a higher voltage with a planar front and a discharge filling the tube during its propagation with an almost constant velocity. However, in comparison to the case at $9 \mathrm{kV}$, for $6 \mathrm{kV}$, the initiation and propagation of the discharge are much slower and the discharge reaches the cathode in $13.3 \mathrm{~ns}$. For $6 \mathrm{kV}$, we note that the discharge is slightly less homogeneous than for $9 \mathrm{kV}$ with a maximum value of electric field off axis of $E=230 \mathrm{kV} / \mathrm{cm}$ and a maximum of electric field on the axis of $E=170 \mathrm{kV} / \mathrm{cm}$. The maximum electron density is also off axis and is $n_{\mathrm{e}}=3.7 \times 10^{14} \mathrm{~cm}^{-3}$ and the maximum electron density on the axis is 2.9 times less. We note that the maximal values of electron density and electric field decrease with the decrease of the applied voltage.

However, these results show that in the range of 6 to $9 \mathrm{kV}$, the discharge propagating in a capillary tube with an inner radius of $R_{\text {tube }}=100 \mu \mathrm{m}$ and permittivity $\varepsilon_{\mathrm{r}}=1$ fills the tube during its propagation and is rather homogeneous behind the discharge front. 


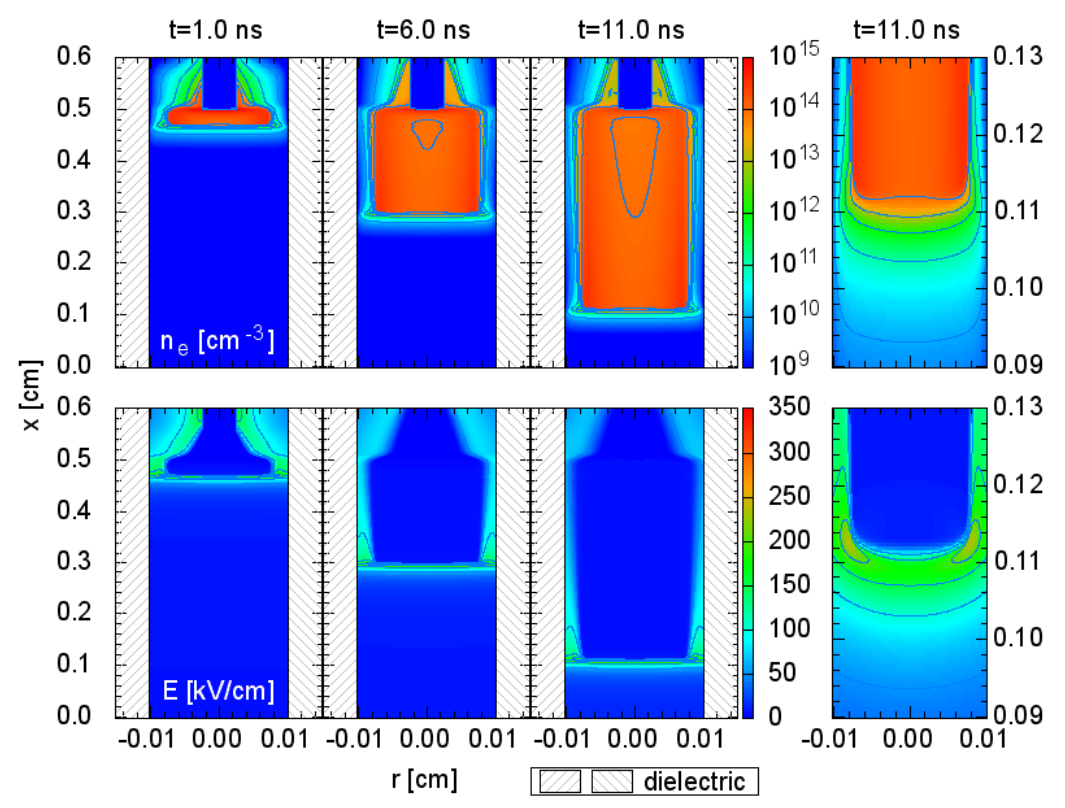

Figure 4. Propagation of the discharge in a capillary tube with $R_{\text {tube }}=100 \mu \mathrm{m}$ and permittivity $\varepsilon_{\mathrm{r}}=1$ for an applied voltage of $6 \mathrm{kV}$. Cross-sectional views of the electron density and the magnitude of the electric field at $t=1,6$, and $11 \mathrm{~ns}$. Last figure of each line shows the discharge front in proportional scale at $t=11 \mathrm{~ns}$.

\subsection{Propagation of the discharge in a dielectric tube with $R_{\text {tube }}=100 \mu \mathrm{m}$ and permittivity $\varepsilon_{\mathrm{r}}>1$}

In this section, we study the influence of the permittivity of the dielectric tube on the discharge structure and dynamics. As a reference, we consider the case of Figure 4 with $\varepsilon_{\mathrm{r}}=1$ and an applied voltage of $6 \mathrm{kV}$. As mentioned in the introduction, we have carried out simulations for three values of $\varepsilon_{\mathrm{r}}=2,5$ and 10 to represent different types of materials. As already discussed in Section 2.2, in this section we have used a value of $\epsilon_{\mathrm{ISG}}=0.1$ in the ISG-1 scheme, to remove small oscillations observed for permittivities $\varepsilon_{\mathrm{r}} \geq 5$. However it was verified that this increase of $\epsilon_{\mathrm{ISG}}$ has a very small influence on the results obtained, as will be shown in Section 3.5. Figure 5 shows the time sequence of the distributions of the electron density and absolute values of the electric field for $\varepsilon_{\mathrm{r}}=5$ (corresponding to glass) and an applied voltage of $6 \mathrm{kV}$. In comparison to Figure 4 , we note that the discharge obtained with $\varepsilon_{\mathrm{r}}=5$ is much less homogeneous and has a tubular structure. The maximum electron density is off axis $\left(n_{e}=5.7 \times 10^{14} \mathrm{~cm}^{-3}\right)$ and is about one order of magnitude higher than the electron density on the axis. The maximum electric field is off axis and is $E=340 \mathrm{kV} / \mathrm{cm}$, while the maximum electric field on the axis is $E=135 \mathrm{kV} / \mathrm{cm}$. Figure 6 shows the axial velocity of the discharge as a function of time for the different values of permittivity of the tube studied in this work. In our simulations, the axial velocity of the discharge is calculated based on the axial movement of the location of the maximum total electric field in the discharge. In the first initiation phase of the discharge, for all cases, the discharge expands in radial and axial directions. For Figure 6, we have only represented the velocity during 
Simulation of the discharge propagation in a capillary tube ...

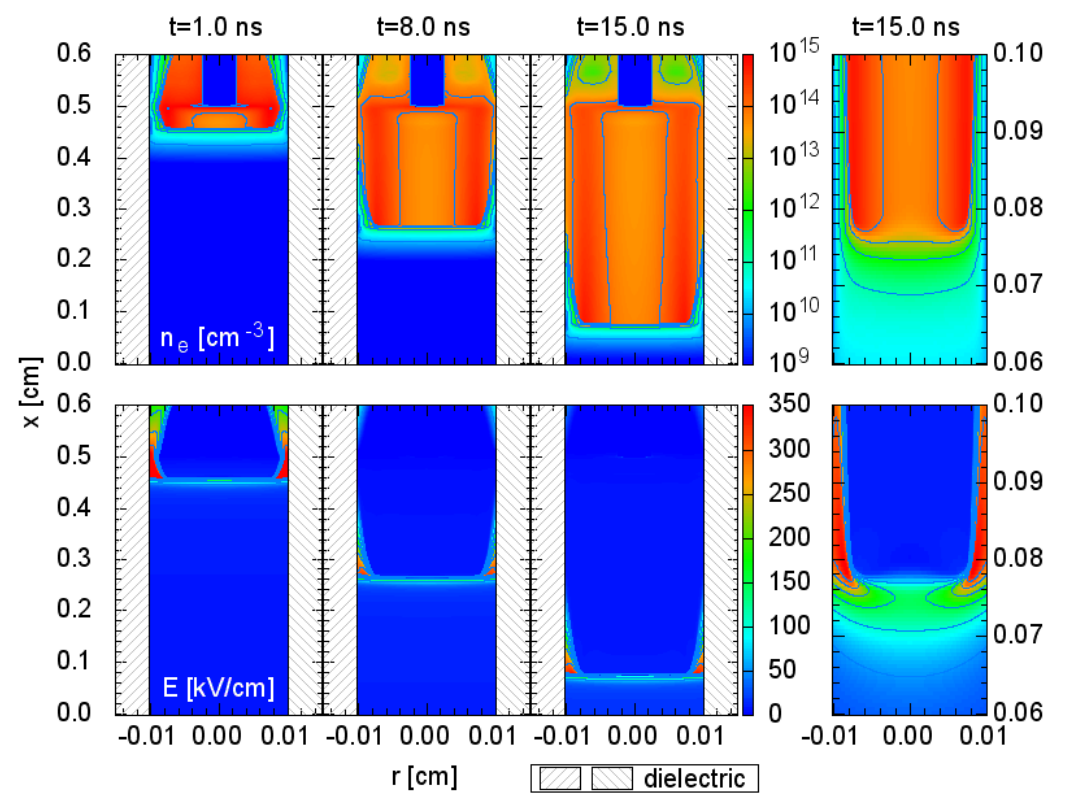

Figure 5. Propagation of the discharge in a capillary tube with $R_{\text {tube }}=100 \mu \mathrm{m}$ and permittivity $\varepsilon_{\mathrm{r}}=5$ for an applied voltage $6 \mathrm{kV}$. Cross-sectional views of the electron density and the magnitude of the electric field at $t=1,8$, and $15 \mathrm{~ns}$. Last figure of each line shows the discharge front in proportional scale at $t=15 \mathrm{~ns}$.

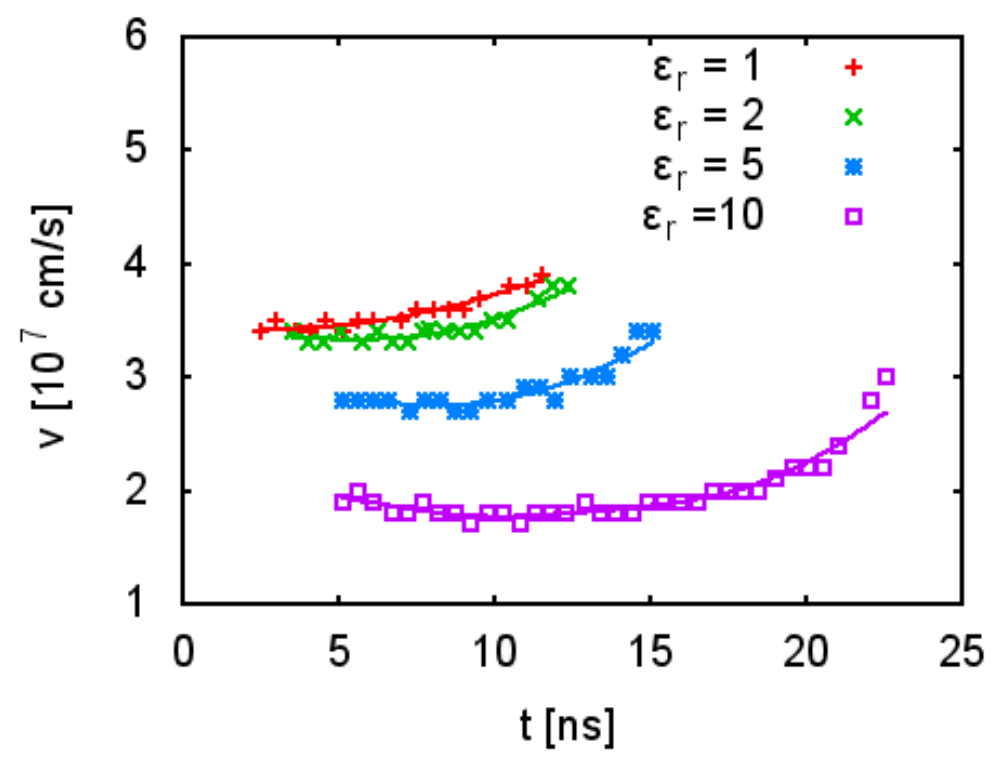

Figure 6. Axial velocity of the discharge front as a function of time for dielectric permittivity constants $\varepsilon_{\mathrm{r}}=1,2,5$ and 10 , a tube with a inner radius of $R_{\text {tube }}=100 \mu \mathrm{m}$ and an applied voltage of $6 \mathrm{kV}$. 
the axial propagation phase towards the cathode and then all curves start after a time corresponding to the initiation phase of the discharge. We note that the value of $\varepsilon_{\mathrm{r}}$ has a small influence on the duration of the initiation phase of the discharge which slightly increases as $\varepsilon_{\mathrm{r}}$ increases. For all values of $\varepsilon_{\mathrm{r}}$, the velocity remains constant during propagation and increases when the discharge approaches the grounded electrode. This corresponds to an electrostatic increase of the total electric field and thus an increase of the region with positive gain of electrons due to the proximity of grounded electrode. Figure 6 shows that the highest velocity is obtained for $\varepsilon_{\mathrm{r}}=1$ and is approximately equal to $3.5 \times 10^{7} \mathrm{~cm} / \mathrm{s}$. In this case the discharge reaches the cathode at $t=13.3 \mathrm{~ns}$. We note that the velocity decreases nonlinearly with the increase of $\varepsilon_{\mathrm{r}}$. For $\varepsilon_{\mathrm{r}}=10$, the velocity is about $1.8 \times 10^{7} \mathrm{~cm} / \mathrm{s}$ and then the discharge reaches the cathode at $t=22.9$ ns. It is interesting to note that an increase of $\varepsilon_{\mathrm{r}}$ by a factor 10 corresponds to a decrease of the velocity of the discharge by about a factor of 2 . For all the values of $\varepsilon_{\mathrm{r}}$, Table 1

\begin{tabular}{c|c|c|c|c|c}
$\varepsilon_{\mathrm{r}}$ & $\begin{array}{c}t_{\mathrm{f}} \\
{[\mathrm{ns}]}\end{array}$ & $\frac{n_{\mathrm{e}, \max }}{n_{\mathrm{e}}(r=0)}$ & $\begin{array}{c}n_{\mathrm{e}, \max } \\
{\left[\mathrm{cm}^{-3}\right]}\end{array}$ & $\begin{array}{c}E_{\max } \\
{[\mathrm{kV} / \mathrm{cm}]}\end{array}$ & $\begin{array}{c}E_{\max }(r=0) \\
{[\mathrm{kV} / \mathrm{cm}]}\end{array}$ \\
\hline 1 & 13.3 & 2.9 & $3.7 \times 10^{14}$ & 230 & 170 \\
2 & 13.7 & 5.5 & $5.0 \times 10^{14}$ & 290 & 160 \\
5 & 17.0 & 11.9 & $5.7 \times 10^{14}$ & 340 & 135 \\
10 & 22.9 & 16.2 & $5.0 \times 10^{14}$ & 390 & 115
\end{tabular}

Table 1. Discharge characteristics for different values of permittivity $\varepsilon_{\mathrm{r}}$, a tube with $R_{\text {tube }}=100 \mu \mathrm{m}$ and an applied voltage of $6 \mathrm{kV} . t_{\mathrm{f}}$ is the time for the discharge to reach the grounded cathode plane. All other quantities are taken at $x=2.5 \mathrm{~mm}$, in the middle of the interelectrode gap. $n_{\mathrm{e}, \max }$ is the maximum electron density and $n_{\mathrm{e}}(r=0)$ is the electron density on the axis. $E_{\max }$ is the maximum total electric field and $E_{\max }(r=0)$ is the maximum electric field on the axis.

shows the time necessary for the discharge to reach the cathode, the maximum electron density and maximum electric field, the ratio of the maximum electron density over the electron density on the axis and the maximum electric field on the axis. All electron density and electric field values of Table 1 are taken at $x=2.5 \mathrm{~mm}$, in the middle of the interelectrode gap. As $\varepsilon_{\mathrm{r}}$ increases, we note that the time necessary for the discharge to reach the cathode increases as are increasing the maximum electron density and electric field. Conversely on the axis, the maximum electric field and electron density decrease as $\varepsilon_{\mathrm{r}}$ increases. Then these results seem to indicate that a more homogeneous discharge front propagates faster than a less homogeneous one. When the discharge front is more homogeneous lower values of maximum electric field and electron density are obtained. It is interesting to note that in the simulations carried out by Georghiou et al. [2005] in air in a configuration which looks close to ours, these authors found that the velocity of the discharge increases as $\varepsilon_{\mathrm{r}}$ increases, which seems to be in disagreement with our results. However, as already mentioned in the introduction, in the work of Georghiou et al. [2005], the discharge is initiated by the needle outside of the tube and there is 
an interaction of the discharge with the curved edges of the tube before the discharge propagates in the tube. Then, it is difficult to compare our results on the influence of $\varepsilon_{\mathrm{r}}$ with those obtained by Georghiou et al. [2005] since the discharge structure is more complex in the case studied by Georghiou et al. [2005] than in our case.

3.4. Propagation of the discharge in a dielectric tube with a radius $R_{\text {tube }} \geq 100 \mu \mathrm{m}$ and permittivity $\varepsilon_{\mathrm{r}}=1$

In this section, we study the influence of the radius of the dielectric tube on the discharge structure and dynamics. As a reference, we consider the case of Figure 3 with a dielectric tube of radius $R_{\text {tube }}=100 \mu \mathrm{m}$, permittivity $\varepsilon_{\mathrm{r}}=1$ and an applied voltage of $9 \mathrm{kV}$. We have carried out simulations for three other inner radii of the tube: $R_{\text {tube }}=300,600$ and $700 \mu \mathrm{m}$. Figure 7 shows the time sequence of the distributions of the electron density

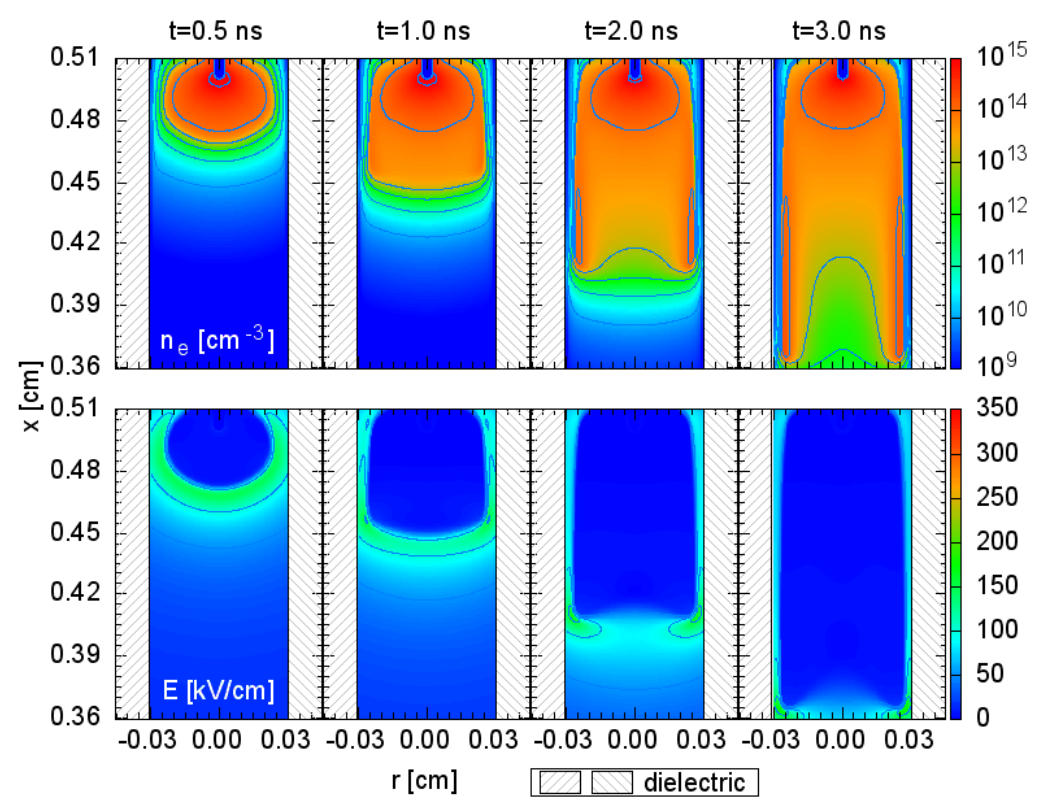

Figure 7. Propagation of the discharge in a capillary tube with $R_{\text {tube }}=300 \mu \mathrm{m}$ and permittivity $\varepsilon_{\mathrm{r}}=1$ for an applied voltage of $9 \mathrm{kV}$. Cross-sectional views of the electron density and the magnitude of the electric field at $t=0.5,1,2$ and 3 ns. All plots are in proportional scale.

and absolute values of the electric field for a tube of radius $R_{\text {tube }}=300 \mu \mathrm{m}$. In the first $0.5 \mathrm{~ns}$, the discharge is initiated and expands in $x$ and $r$ directions. At $t=0.5 \mathrm{~ns}$, we clearly see that the presence of the tube limits the radial expansion of the discharge. At time $t=1 \mathrm{~ns}$, a local maximum of electric field appears close to the tube surface, and the discharge starts propagating axially. At $t=3 \mathrm{~ns}$, the obtained discharge has a very pronounced tubular structure. The maximum electron density is off axis and is $2.2 \times 10^{14} \mathrm{~cm}^{-3}$ which is about two orders of magnitude higher than the electron density on the axis. The maximum electric field is out of axis and is $E=180 \mathrm{kV} / \mathrm{cm}$, while the maximum electric field on the axis is $E=80 \mathrm{kV} / \mathrm{cm}$. It is important to mention that in this work, we simulate axisymmetric discharges and then the significant radial 
inhomogeneities of the discharge on Figure 7 may correspond to a 3D discharge structure. However, first experimental results with a glass tube of various radii seem to confirm the tubular structure of the discharge [LeDelliou et al., 2010]. The detailed comparison with experiment will be presented in a separate dedicated paper. Figure 8 shows the axial

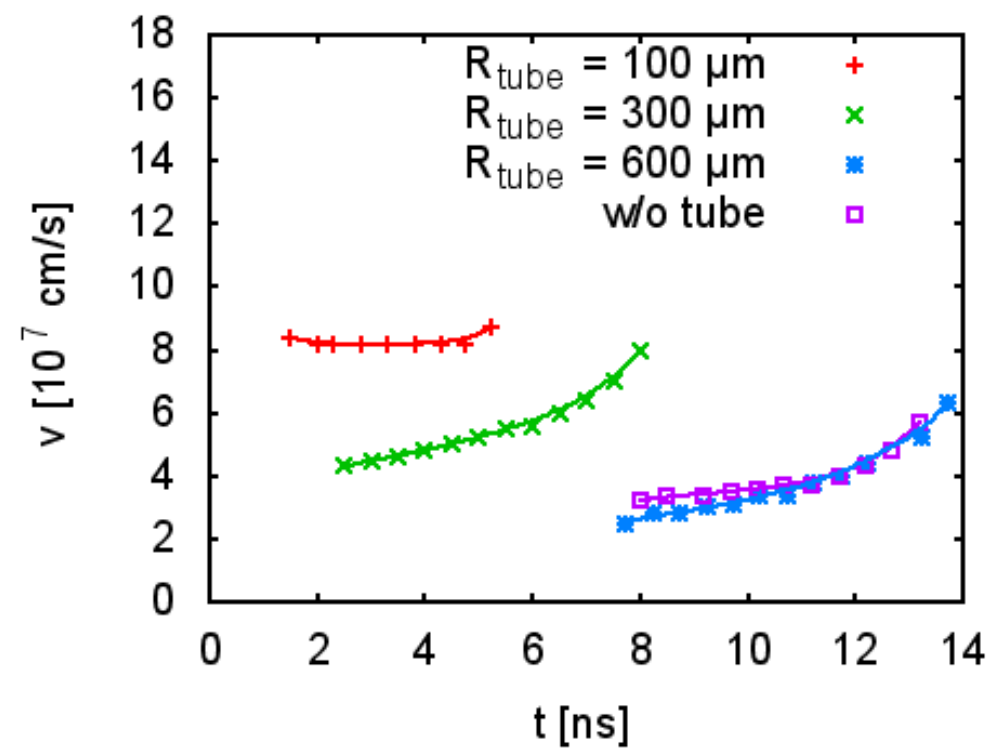

Figure 8. Axial velocity of the discharge front as a function of time without dielectric tube and for tubes of inner radius $R_{\text {tube }}=100,300$ and $600 \mu \mathrm{m}, \varepsilon_{\mathrm{r}}=1$ and an applied voltage of $9 \mathrm{kV}$.

velocity of the discharge as a function of time for the case without dielectric (Figure 2) and for three radii of the tube: $R_{\text {tube }}=100,300$ and $600 \mu \mathrm{m}$. The results obtained for $R_{\text {tube }}=700 \mu \mathrm{m}$ are very close to those obtained for the case without dielectric tube and are not shown here for sake of clarity. We note that the highest velocity of the discharge is obtained for $R_{\text {tube }}=100 \mu \mathrm{m}$ and the velocity decreases as the radius of the tube increases to converge towards the velocity of the discharge without a dielectric tube. With a dielectric tube of $R_{\text {tube }}=100 \mu \mathrm{m}$, the mean axial velocity is between $2-4$ times larger than the velocity without dielectric tube. It is interesting to note that for $R_{\text {tube }}=100 \mu \mathrm{m}$, the velocity is constant during the whole propagation phase and increases only at the end when the discharge is close to the cathode. For larger radii, we note that the velocity of the discharge slightly increases during all its axial propagation. Finally, as already mentioned in Figure 6, the time of start of the different curves on Figure 8 gives an information on the duration of the first expansion phase of the discharge and the transition to the axial propagation. In Figure 8, this time also shows the interaction of the discharge with the tube. As expected for smaller radii, the start of the axial propagation is earlier than for larger radii. For all the values of the tube radius, Table 2 shows the time necessary for the discharge to reach the cathode, the maximum electron density and maximum electric field, the ratio of the maximum electron density over the electron density on the axis and the maximum electric field 
Simulation of the discharge propagation in a capillary tube ...

\begin{tabular}{c|c|c|c|c|c}
$R_{\text {tube }}$ & $\begin{array}{c}t_{\mathrm{f}} \\
{[\mathrm{ns}]}\end{array}$ & $\begin{array}{c}n_{\mathrm{e}, \max } \\
n_{\mathrm{e}}(r=0)\end{array}$ & $\begin{array}{c}n_{\mathrm{e}, \max } \\
{\left[\mathrm{cm}^{-3}\right]}\end{array}$ & $\begin{array}{c}E_{\max } \\
{[\mathrm{kV} / \mathrm{cm}]}\end{array}$ & $\begin{array}{c}E_{\max }(r=0) \\
{[\mathrm{kV} / \mathrm{cm}]}\end{array}$ \\
\hline $100 \mu \mathrm{m}$ & 5.7 & 2.8 & $5.5 \times 10^{14}$ & 300 & 220 \\
$300 \mu \mathrm{m}$ & 8.4 & 100 & $2.2 \times 10^{14}$ & 180 & 80 \\
$600 \mu \mathrm{m}$ & 14.2 & 1000 & $1.6 \times 10^{14}$ & 140 & 50 \\
$700 \mu \mathrm{m}$ & 13.6 & 1 & $3.2 \times 10^{13}$ & 110 & 110 \\
w/o tube & 13.8 & 1 & $3.5 \times 10^{13}$ & 110 & 110
\end{tabular}

Table 2. Discharge characteristics for different radii $R_{\text {tube }}$ of the tube, $\varepsilon_{\mathrm{r}}=1$ and an applied voltage of $9 \mathrm{kV} . t_{\mathrm{f}}$ is the time for the discharge to reach the grounded cathode plane. All other quantities are taken at $x=2.5 \mathrm{~mm}$, in the middle of the interelectrode gap. $n_{\mathrm{e}, \max }$ is the maximum electron density and $n_{\mathrm{e}}(r=0)$ is the electron density on the axis. $E_{\max }$ is the maximum total electric field and $E_{\max }(r=0)$ is the maximum electric field on the axis.

on the axis. As the radius of the tube increases from $R_{\text {tube }}=100 \mu \mathrm{m}$ to $600 \mu \mathrm{m}$, we note that the time necessary for the discharge to reach the cathode increases whereas the maximum electron density and electric field decrease. For higher values of the radius, the influence of the tube becomes negligible and the discharge has the same characteristics as the one calculated without tube. As already mentioned in Section 3.2, it is interesting to note that for $R_{\text {tube }}=100 \mu \mathrm{m}$, the maximum electron density and electric field are respectively 16 and 3 times higher than those obtained without tube. Table 2 also shows that the ratio of the maximum electron density to the electron density on the axis strongly depends on the tube radius. We note that the discharge propagates on the axis for $R_{\text {tube }} \geq 700 \mu \mathrm{m}$ and fills the tube homogeneously during its propagation for a small radius of $R_{\text {tube }}=100 \mu \mathrm{m}$. For intermediate values of radius, the discharge has a very pronounced tubular structure. As already observed in Table 1, the results of Table 2 also show that in capillary tubes, a discharge with a more homogeneous discharge front propagates faster than a less homogeneous one.

Finally, based on the results obtained in this section and the preceding ones for applied voltages of 6 and $9 \mathrm{kV}$, we propose to compare the influence of the radius of the tube and of the value of $\varepsilon_{\mathrm{r}}$ on the discharge structure and dynamics. First, it is important to mention that in experiments it is difficult to study separately the effects of geometry and $\varepsilon_{\mathrm{r}}$. In our simulations, we have used $\varepsilon_{\mathrm{r}}=1$ as a reference case to study only the influence of the geometrical constraint of the tube on the discharge. In previous sections, for $6 \mathrm{kV}$ and a constant tube radius of $100 \mu \mathrm{m}$, we have shown that an increase of $\varepsilon_{\mathrm{r}}$ by a factor 10 leads to a decrease of the discharge velocity by almost a factor 2 . Then, for $9 \mathrm{kV}$ and $\varepsilon_{\mathrm{r}}=1$, we have shown that an increase of the tube radius by a factor 6 corresponds to a decrease of the discharge velocity by a factor 4 in the beginning of propagation to a factor 1.3 at the end. Based on these results, it seems that the geometrical constraint of the cylindrical tube on the discharge has slightly more influence on the discharge structure and dynamics than the value of $\varepsilon_{\mathrm{r}}$. 


\subsection{Influence of photoemission}

For all the results presented in previous sections, the only secondary process at the dielectric surface is secondary emission due to ion bombardment. This secondary process requires to have a sufficient density of ions close to the surface. As already mentioned in Section 2.2, we have tested different values of the secondary emission coefficient in the range $0.001-0.1$ and no visible differences on the discharge propagation velocity or maximum electric field have been observed. In our simulations, we have verified that secondary emission is important close to the surface behind the discharge front. In

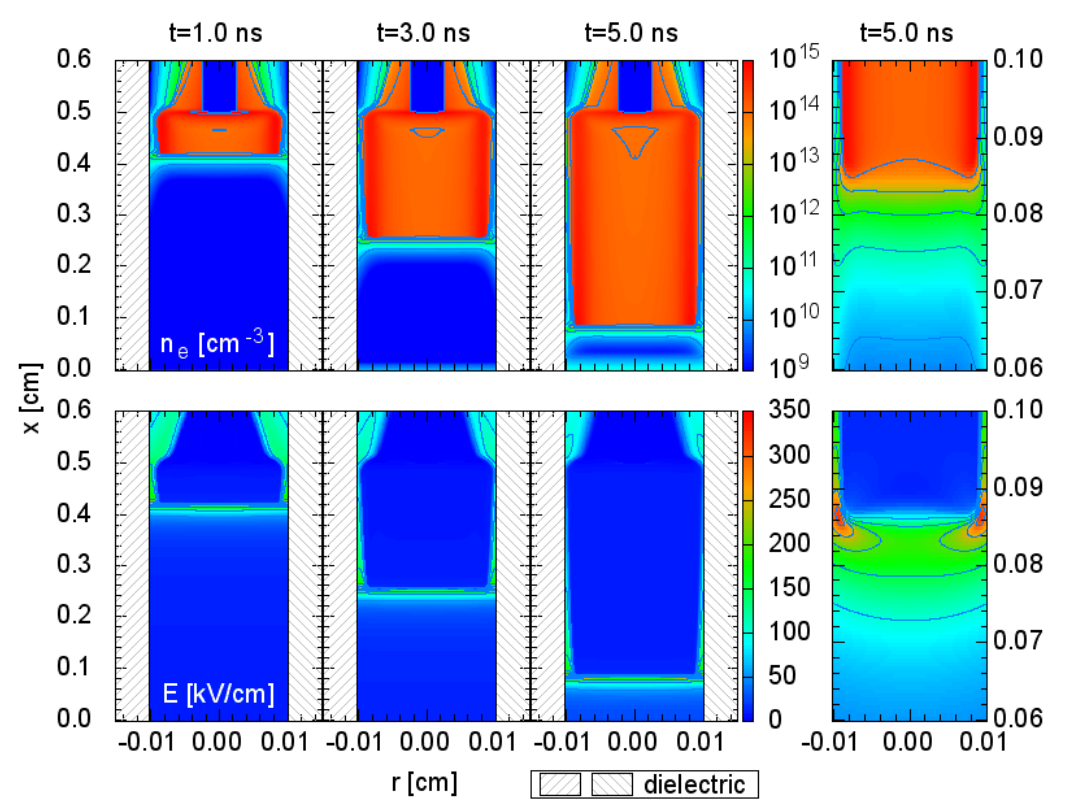

Figure 9. Propagation of the discharge in a capillary tube with $R_{\text {tube }}=100 \mu \mathrm{m}$ and permittivity $\varepsilon_{\mathrm{r}}=1$ for an applied voltage $9 \mathrm{kV}$. Photoemission source term is included with coefficient $\gamma_{\text {photoem }}=5.0 \times 10^{-4}$. Cross-sectional views of the electron density and the magnitude of the electric field at $t=1,3$, and 5 ns. Last figure of each line shows the discharge front in proportional scale at $t=5 \mathrm{~ns}$.

this section, we have included the photoemission source term, which has a very different dynamics compared to secondary emission by ion bombardement. Indeed, photoemission produces very rapidly (as it is due to radiation) secondary electrons at the surface ahead of the discharge front. As mentioned in Section 2.2, we have considered three values for the photoemission coefficient $\gamma_{\text {photoem }}=5 \times 10^{-4}, 5 \times 10^{-3}, 1 \times 10^{-1}$. Furthermore, to discuss about the influence of the value of $\epsilon_{\mathrm{ISG}}$ in Kulikovsky scheme, in this section we have used a value of $\epsilon_{\mathrm{ISG}}=0.1$ to compare with results obtained in sections 3.1, 3.2 and 3.4 where a value of $\epsilon_{\mathrm{ISG}}=0.01$ was used. Figure 9 shows the time sequence of the distributions of the electron density and absolute values of the electric field for a tube of radius $R_{\text {tube }}=100 \mu \mathrm{m}, \varepsilon_{\mathrm{r}}=1$, an applied voltage of $9 \mathrm{kV}$ and a photoemission coefficient of $\gamma_{\text {photoem }}=5 \times 10^{-4}$. On the proportional figure of electron density at $t=5 \mathrm{~ns}$, the increase of electron density close to the tube in front of the discharge is clearly seen. In comparison to the case without photoemission (Figure 3), we note that the discharge is 
slightly less homogeneous when photoemission is included. Table 3 shows for an applied

\begin{tabular}{c|c|c|c|c|c}
$\gamma_{\text {photoem }}$ & $\begin{array}{c}t_{\mathrm{f}} \\
{[\mathrm{ns}]}\end{array}$ & $\frac{n_{\mathrm{e}, \max }}{n_{\mathrm{e}}(r=0)}$ & $\begin{array}{c}n_{\mathrm{e}, \max } \\
{\left[\mathrm{cm}^{-3}\right]}\end{array}$ & $\begin{array}{c}E_{\max } \\
{[\mathrm{kV} / \mathrm{cm}]}\end{array}$ & $\begin{array}{c}E_{\max }(r=0) \\
{[\mathrm{kV} / \mathrm{cm}]}\end{array}$ \\
\hline 0 & 5.7 & 2.5 & $5.6 \cdot 10^{14}$ & 310 & 220 \\
$5 \times 10^{-4}$ & 5.7 & 6.6 & $7.4 \cdot 10^{14}$ & 315 & 185 \\
$5 \times 10^{-3}$ & 5.5 & 90 & $8.0 \cdot 10^{14}$ & 285 & 130 \\
$1 \times 10^{-1}$ & 6.3 & 1000 & $4.1 \cdot 10^{14}$ & 200 & 90
\end{tabular}

Table 3. Discharge characteristics for different values of the photoemission coefficient $\gamma_{\text {photoem }}$, for a tube of radius $100 \mu \mathrm{m}, \varepsilon_{\mathrm{r}}=1$ and applied voltage of $9 \mathrm{kV} . t_{\mathrm{f}}$ is the time for the discharge to reach the grounded cathode plane. All other quantities are taken at $x=2.5 \mathrm{~mm}$, in the middle of the interelectrode gap. $n_{\mathrm{e}, \max }$ is the maximum electron density and $n_{\mathrm{e}}(r=0)$ is the electron density on the axis. $E_{\max }$ is the maximum total electric field and $E_{\max }(r=0)$ is the maximum electric field on the axis.

voltage of $9 \mathrm{kV}$, the influence of the value of photoemission coefficient on discharge characteristics. We note that photoemission has a negligible influence on the time $t_{\mathrm{f}}$ for the discharge to reach the cathode and maximum electric field for $\gamma_{\text {photoem }}=0$ to $5 \times 10^{-3}$. For a very high value of $\gamma_{\text {photoem }}=1 \times 10^{-1}, t_{\mathrm{f}}$ slightly increases and maximum electric field slightly decreases. However, the discharge structure is significantly affected

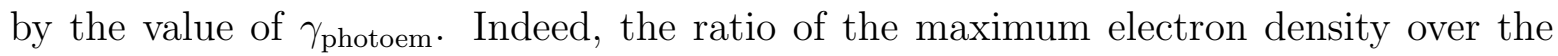
electron density on the axis increases significantly with increasing $\gamma_{\text {photoem }}$. This clearly shows that with a higher $\gamma_{\text {photoem }}$, more seed electrons are produced close to the surface and then the discharge propagates more easily at the surface than in the volume and becomes non homogeneous. For $\gamma_{\text {photoem }}=5 \times 10^{-4}$ to $5 \times 10^{-3}$, it is interesting to note that the different discharge structure has no influence on the velocity of the discharge. Only for a very high value $\gamma_{\text {photoem }}=1 \times 10^{-1}$, we have a decrease of the velocity of the discharge due to its significant inhomogeneity, as already observed in previous sections. Finally, we propose to study the influence of $\epsilon_{\mathrm{ISG}}$, by comparing for the case $9 \mathrm{kV}$, $R_{\text {tube }}=100 \mu \mathrm{m}, \varepsilon_{\mathrm{r}}=1$ and $\gamma_{\text {photoem }}=0$, the results obtained in Table $2\left(\epsilon_{\mathrm{ISG}}=0.01\right)$ and Table $3\left(\epsilon_{\mathrm{ISG}}=0.1\right)$. We note that the change of value of $\epsilon_{\mathrm{ISG}}$ has a negligible influence on the time for the discharge to reach the cathode, and only a very small influence on electron density and electric field.

\section{Conclusions}

In this work, we present simulations of the structure and dynamics of a discharge in air at atmospheric pressure initiated by a needle set in a dielectric capillary tube. A constant voltage has been assumed for all simulations and we have studied the $6-9 \mathrm{kV}$ range. As a reference, we have first studied the discharge in the needle-to-plane configuration without dielectric tube. In this case after a first axial and radial expansion phase around the needle tip, the discharge propagates axially with a rather constant 
velocity. The discharge radius is about $600 \mu \mathrm{m}$, which is about the radius of the largest capillary tube we have studied in this work. Then we have studied the influence of the radius of the tube and the value of the permittivity $\varepsilon_{\mathrm{r}}$ of the dielectric on the discharge. In experiments, it is difficult to study separately the effects of geometry and $\varepsilon_{\mathrm{r}}$. In our simulations, we have used $\varepsilon_{\mathrm{r}}=1$ as a reference case to study only the influence of the cylindrical constraint of the tube on the discharge. For a tube radius of $100 \mu \mathrm{m}$, we have shown that after the initiation phase the discharge propagates in the tube with a rather planar discharge front. The discharge fills the tube during its propagation and is rather homogeneous behind the discharge front. In comparison to the case without dielectric tube, we note that the initiation and propagation phases are much faster with a capillary tube. The maximum electron density and electric field are both higher with the tube than without and then, the reactivity of the discharge is expected to be higher with the tube than without. The homogeneous and chemically active discharge obtained for a tube radius of $100 \mu \mathrm{m}$ is particularly interesting for different types of applications as for example for the treatment of polluted air flows with plasma-catalyst coupling. Then we have studied the influence of $\varepsilon_{\mathrm{r}}$ on the discharge for a constant tube radius of $100 \mu \mathrm{m}$. We have shown that an increase of $\varepsilon_{\mathrm{r}}$ by a factor 10 leads to a decrease of the discharge velocity by about a factor of 2 .

Then we have studied for $\varepsilon_{\mathrm{r}}=1$, the influence of the tube radius on the discharge. We have shown that an increase of the tube radius by a factor 6 corresponds to a decrease of the discharge velocity by a factor 4 in the beginning of propagation to a factor 1.3 at the end. It is interesting to note that the increase of $\varepsilon_{\mathrm{r}}$ and of the tube radius both decrease the discharge velocity. However, based on our results, it seems that the geometrical constraint of the cylindrical tube on the discharge has slightly more influence on the discharge structure than the value of $\varepsilon_{\mathrm{r}}$.

For a tube radius of $100 \mu \mathrm{m}$, we have shown that as $\varepsilon_{\mathrm{r}}$ increases, the discharge structure becomes tubular with an increase of maximum electric field and electron density values close to the tube surface and a decrease of the electric field and electron density on the axis. As discharge velocity decreases as $\varepsilon_{\mathrm{r}}$ increases, our results show that a discharge with a more homogeneous front propagates faster in the tube than a less homogeneous one.

For $\varepsilon_{\mathrm{r}}=1$ when the tube radius is varied, we have shown that for a small tube radius of $R_{\text {tube }}=100 \mu \mathrm{m}$, the discharge fills the tube during its propagation and that for $R_{\text {tube }} \geq 700 \mu \mathrm{m}$, the tube has no influence on the discharge structure. For intermediate values of tube radius, the discharge has a very pronounced tubular structure. We note that the radius of the tube has an influence on the maximum electric field. Indeed, during the first expansion phase the total electric field around the sphere-shaped plasma is decreasing. The discharge starts to propagate when the expansion phase is restricted by the tube and maximum of electric field moves close to the tube surface. A smaller radius corresponds to a shorter expansion phase and then a higher maximum total electric field when the discharge starts to propagate axially.

Then, as expected, we have shown that photoemission increases the number of sec- 
ondary electrons close to the dielectric surface and then promotes the propagation of the discharge on the surface. As the photoemission coefficient in air is poorly known, we have considered a wide range of values from $5 \times 10^{-4}$ to $1 \times 10^{-1}$. We note that when the photoemission coefficient increases, the tubular structure of the discharge becomes more pronounced. However, the time to reach the cathode remains constant for a wide range of values of the photoemission coefficient and decreases only for very high values of the photoemission coefficient.

It is important to note that, in this work, we simulate axisymmetric discharges and then significant radial inhomogeneities of the discharge obtained for example for $\varepsilon_{\mathrm{r}}=1$ and $300 \leq R_{\text {tube }} \leq 600 \mu \mathrm{m}$ may correspond to a 3D discharge structure. However, first experimental results for glass tubes of various radii seem to confirm the tubular structure of the discharge [LeDelliou et al., 2010]. In this work, we have assumed that the applied voltage is constant and that no surface charges are deposited on the dielectric tube by previous discharges that occurred before the simulated one. To compare with experimental results, it would be important to take into account the real shape of the pulse voltage and to study the influence of surface charges deposited on the dielectric surface on the discharge structure and dynamics. The detailed comparison with experimental results will be presented in a dedicated paper.

\section{Acknowledgments}

The authors thank the Agence Nationale de la Recherche for its support of the ALVEOPLAS project (Grant No. ANR-08-BLAN-0159-01).

\section{References}

Allen, N. L., and M. Mikropoulos (1999), Streamer propagation along insulating surfaces, IEEE Transactions on Dielectrics and Electrical Insulation, 6(3), 357-362.

Amestoy, P., I. Duff, J. L'Excellent, and J. Koster (2001), A fully asynchronous multifrontal solver using distributed dynamic scheduling, SIAM Journal of Matrix Analysis and Applications, 23, 15-41.

Babaeva, N. Y., and M. J. Kushner (2009), Effect of inhomogeneities on streamer propagation: I. intersection with isolated bubbles and particles, Plasma Sources Sci. Technol., 18(3), doi:10.1088/0963-0252/18/3/035009.

Babaeva, N. Y., and G. V. Naidis (1996), Two-dimensional modelling of positive streamer dynamics in non-uniform electric fields in air, J. Phys. D: Appl. Phys., 29, 2423-2431, doi:10.1088/0022-3727/29/9/029.

Babaeva, N. Y., A. N. Bhoj, and M. J. Kushner (2006), Streamer dynamics in gases containing dust particles, Plasma Sources Sci. Technol., 15, 591-602, doi: 10.1088/0963-0252/15/4/001. 
Becker, K., K. Schoenbach, and J. Eden (2006), Microplasmas and applications, J. Phys. D:Appl. Phys., 39, R55-R70, doi:10.1088/0022-3727/39/3/R01.

Bhoj, A. N., and M. J. Kushner (2008), Repetitively pulsed atmospheric pressure discharge treatment of rough polymer surfaces: I. humid air discharges, Plasma Sources Sci. Technol., $17(3)$, doi:10.1088/0963-0252/17/3/035024.

Blin-Simiand, N., P. Tardiveau, A. Risacher, F. Jorand, and S. Pasquiers (2005), Removal of 2-heptanone by dielectric barrier discharges - The effect of a catalyst support, Plasma Process. Polym., 2(3), 256-262.

Bourdon, A., V. Pasko, N. Liu, S. Célestin, P. Ségur, and E. Marode (2007), Efficient models for photoionization produced by non-thermal gas discharges in air based on radiative transfer and Helmholtz equations, Plasma Sources Sci. Technol., 16(3), 656-678, doi:10.1088/0963-0252/16/3/026.

Brandeburg, R., Z. Navrátil, J. Jánský, P. Sťahel, D. Trunec, and H.-E. Wagner (2009), The transition between different modes of barrier discharges at atmospheric pressure, J. Phys. D: Appl. Phys., 42(8), doi:10.1088/0022-3727/42/8/085208.

Célestin, S., Z. Bonaventura, B. Zeghondy, A. Bourdon, and P. Ségur (2009), The use of the ghost fluid method for Poisson's equation to simulate streamer propagation in point-to-plane and point-to-point geometries, J. Phys. D-Appl. Phys., 42(6), doi: 10.1088/0022-3727/42/6/065203.

Djermoune, D., E. Marode, and P. Ségur (1995), Two dimensional modelling of a streamer induced discharge, in Proc. 22th Int. Conf. on Phenomena in Ionized Gases (Hoboken, USA), pp. 33-34.

Eichwald, O., O. Ducasse, D. Dubois, A. Abahazem, N. Merbahi, M. Benhenni, and M. Yousfi (2008), Experimental analysis and modelling of positive streamer in air: towards an estimation of $\mathrm{O}$ and $\mathrm{N}$ radical production, J. Phys. D: Appl. Phys., 41 (23), doi:10.1088/0022-3727/41/23/234002.

Georghiou, G. E., R. Morrow, and A. C. Metaxas (2001), The effect of photoemission on the streamer developement and propagation in short uniform gaps, J. Phys. D: Appl. Phys., 34(2), 200-208, doi:10.1088/0022-3727/34/2/308.

Georghiou, G. E., A. P. Papadakis, R. Morrow, and A. C. Metaxas (2005), Numerical modelling of atmospheric pressure gas discharges leading to plasma production, $J$. Phys. D: Appl. Phys., 38(20), R303-R328, doi:10.1088/0022-3727/38/20/R01.

Gibalov, V., and G. Pietsch (2000), The development of dielectric barrier discharges in gas gaps and on surfaces, J. Phys. D: Appl. Phys., 33, 2618-2636.

Guaitella, O., F. Thevenet, C. Guillard, and A. Rousseau (2006), Dynamic of the plasma current amplitude in a barrier discharge: influence of photocatalytic material, J. Phys. D: Appl. Phys., 39(14), 2964-2972, doi:10.1088/0022-3727/39/14/015.

Hallac, A., G. E. Georghiou, and A. C. Metaxas (2003), Secondary emission effects on streamer branching in transient non-uniform short-gap discharges, J. Phys. D: Appl. Phys., 36, 2498-2509, doi:10.1088/0022-3727/36/20/011. 
Hammer, T. (2002), Non-thermal plasma application to the abatment of noxious emissions in automotive exhaust gases, Plasma Sources Sci. Technol., 11, A196.

Hensel, K. (2009), Microdischarges in ceramic foams and honeycombs, European Physical Journal D, 54(2), 141-148, doi:10.1140/epjd/e2009-00073-1.

Hensel, K., V. Martisovit, Z. Machala, M. Janda, M. Lestinsky, P. Tardiveau, and A. Mizuno (2007), Electrical and optical properties of AC microdischarges in porous ceramics, Plasma Process. Polym., 4(7-8), 682-693, doi:10.1002/ppap.200700022.

Kim, H. H. (2004), Non-thermal plasma processing for air pollution control : a historical review, current issues and future prospects, Plasma Process. Polym., 1(2), 91-110.

Kulikovsky, A. A. (1995), A more accurate Scharfetter-Gummel algorithm of electron transport for semiconductor and gas discharge simulation, J. Comput. Phys., 119, $149-155$.

Kulikovsky, A. A. (1997), Positive streamer between parallel plate electrodes in atmospheric pressure air, J. Phys. D: Appl. Phys., 30, 441-450.

Kulikovsky, A. A. (1998), Positive streamer in a weak field in air: A moving avalancheto-streamer transition, Phys. Rev. E, 57, 7066-7074, doi:10.1103/PhysRevE.57.7066.

LeDelliou, P., P. Tardiveau, P. Jeanney, G. Bauville, F. Jorand, and S. Pasquiers (2010), Time resolved propagation of a positive capillary discharge in air at atmospheric pressure, submitted to the XVIII International Conference on Gas Discharges and Their Applications (GD2010).

Liu, N. Y., S. Célestin, A. Bourdon, V. P. Pasko, P. Ségur, and E. Marode (2007), Application of photoionization models based on radiative transfer and the Helmhotz equations to studies of streamers in weak electric fields, Appl. Phys. Lett., 91, 211,501, doi:10.1063/1.2816906.

Lu, X., and M. Laroussi (2006), Dynamics of an atmospheric pressure plasma plume generated by submicrosecond voltage pulses, J. Appl. Phys., 100(6), doi: 10.1063/1.2349475.

Morrow, R., and J. J. Lowke (1997), Streamer propagation in air, J. Phys. D: Appl. Phys., 30, 614-627.

Odrobina, I., and M. Cernak (1991), Numerical modeling of the streamer-cathode interaction in a short positive point-plane corona gap, Czechoslovak Journal of Physics, 42(3), 303-315.

Pasquiers, S. (2004), Removal of pollutants by plasma catalytic processes, Eur. Phys. J. Appl. Phys., 28, 319-324.

Potin, J. (2001), Phd thesis, Ph.D. thesis, University Paul Sabatier, Toulouse, France.

Sands, B. L., B. N. Ganguly, and K. Tachibana (2008), A streamer-like atmospheric pressure plasma jet, Appl. Phys. Lett., 92(15), doi:10.1063/1.2909084.

Sato, S., K. Hensel, H. Hayashi, K. Takashima, and A. Mizuno (2009), Honeycomb discharge for diesel exhaust cleaning, J. Electrost., 67(2-3), 77-83, doi: 10.1016/j.elstat.2009.02.004. 
Shi, J., F. Zhong, J. Zhang, D. W. Liu, and M. G. Kong (2008), A hypersonic plasma bullet train traveling in an atmospheric dielectric-barrier discharge jet, Phys. Plasmas, 15(1), doi:10.1063/1.2828551.

Sobota, A., A. Lebouvier, N. J. Kramer, E. M. van Veldhuizen, W. W. Stoffels, F. Manders, and M. Haverlag (2009), Speed of streamers in argon over a flat surface of a dielectric, J. Phys. D: Appl. Phys., 42(1), doi:10.1088/0022-3727/42/1/015211.

Tan, B., N. Allen, and H. Rodrigo (2007), Progression of positive corona on cylindrical insulating surfaces part i: influence of dielectric material, IEEE Transactions on Dielectrics and Electrical Insulation, 14(1), 111-118.

Tardiveau, P., N. Moreau, S. Bentaleb, C. Postel, and S. Pasquiers (2009), Diffuse mode and diffuse-to-filamentary transition in a high pressure nanosecond scale corona discharge under high voltage, J. Phys. D: Appl. Phys, 42(17), doi:10.1088/0022$3727 / 42 / 17 / 175202$.

Teschke, M., J. Kedzierski, E. G. Finantu-Dinu, D. Korzec, and J. Engemann (2005), High-speed photographs of a dielectric barrier atmospheric pressure plasma jet, IEEE Trans. Plasma Sci., 33(2, Part 1), 310-311, doi:10.1109/TPS.2005.845377.

Unfer, T., and J. P. Boeuf (2009), Modelling of nanosecond surface discharge actuator, J. Phys. D: Appl. Phys., 42(19), doi:10.1088/0022-3727/42/19/194017.

Walsh, J. L., F. Iza, N. B. Janson, V. J. Law, and M. G. Kong (2010), Three distinct modes in a cold atmospheric pressure plasma jet, J. Phys D: Appl. Phys., 43(7), doi:10.1088/0022-3727/43/7/075201. 